\title{
O processo saúde-doença e a velhice: reflexões acerca do normal e do patológico
}

\author{
The health-disease process and old age: reflections about the normal and pathological \\ El proceso de salud-enfermedad y la vejez: reflexiones sobre lo normal y patológico
}

Recebido: 14/01/2021 | Revisado: 17/01/2021 | Aceito: 19/01/2021 | Publicado: 24/01/2021

Nidia Farias Fernandes Martins

ORCID: https://orcid.org/0000-0001-5652-1110

Universidade Federal do Rio Grande, Brasil

E-mail: nidiaffmartins@gmail.com

Daiane Porto Gautério Abreu

ORCID: https://orcid.org/0000-0002-1125-4693

Universidade Federal do Rio Grande, Brasil

E-mail: daianeportoabreu@gmail.com

Rosemary Silva da Silveira

ORCID: https://orcid.org/0000-0003-0671-0022

Universidade Federal do Rio Grande, Brasil

E-mail: rosemaryssilveira@gmail.com

Juliana Piveta de Lima

ORCID: https://orcid.org/0000-0002-2703-9189

Universidade Federal do Rio Grande, Brasil

E-mail: julianapivettal@hotmail.com

Eliel de Oliveira Bandeira

ORCID: https://orcid.org/0000-0003-1038-1612

Universidade Federal do Rio Grande, Brasil

E-mail: bandeira.eliel@hotmail.com

Carolina de Souza Carvalho Serpa Santos

ORCID: https://orcid.org/0000-0002-6847-2616

Universidade Federal do Rio Grande, Brasil

E-mail: carolinaenfermeiraa@gmail.com

\section{Resumo}

Este artigo tem como objetivo refletir acerca do processo saúde-doença na velhice, na perspectiva do normal e do patológico. Trata-se de um ensaio teórico-reflexivo, com base filosófica, que se deu por meio da obra "O normal e o patológico", do filósofo e médico francês Georges Canguilhem. Para embasar a reflexão e a discussão, foram utilizadas publicações científicas acerca das temáticas sobre envelhecimento humano, velhice, processo saúde-doença no envelhecimento e na velhice, normal e patológico, e algumas políticas públicas. A reflexão propõe dois capítulos: "o processo saúde-doença na velhice: o que é normal e o que é patológico?" e "envelhecimento e velhice no plano das políticas públicas de saúde". A quebra de paradigmas referentes ao envelhecimento e a velhice ainda é incipiente, tanto na sociedade quanto nas políticas públicas de saúde. Envelhecer é algo complexo, não cabendo fixar limites ou fronteiras entre essas duas vertentes, ou entre a saúde e a doença.

Palavras-chave: Processo saúde-doença; Envelhecimento; Idoso; Políticas públicas de saúde.

\begin{abstract}
This article aims to reflect on the health-disease process in old age, from the perspective of normal and pathological. It is a theoretical-reflective essay, with a philosophical basis, which took place through the work "O normal e o patológico", by the French philosopher and physician Georges Canguilhem. To support the reflection and discussion, scientific publications were used on the themes of human aging, old age, health-disease process in aging and old age, normal and pathological, and some public policies. The reflection proposes two chapters: "the health-disease process in old age: what is normal and what is pathological?" and "aging and old age in terms of public health policies". The paradigm shift regarding aging and old age is still incipient, both in society and in public health policies. Aging is complex, and there is no need to set limits or boundaries between these two aspects, or between health and disease.
\end{abstract}

Keywords: Health-disease process; Aging; Aged; Public health policy.

\section{Resumen}

Este artículo tiene como objetivo reflexionar sobre el proceso salud-enfermedad en la vejez, desde la perspectiva de lo normal y lo patológico. Se trata de un ensayo teórico-reflexivo, de base filosófica, que se realizó a través de la obra "O normal e o patológico", del filósofo y médico francés Georges Canguilhem. Para apoyar la reflexión y la discusión se utilizaron publicaciones científicas sobre los temas de envejecimiento humano, vejez, proceso salud-enfermedad en 
el envejecimiento y la vejez, normal y patológico, y algunas políticas públicas. La reflexión propone dos capítulos: "El proceso salud-enfermedad en la vejez: ¿qué es normal y qué es patológico?" y "envejecimiento y vejez en términos de políticas de salud pública". El cambio de paradigma sobre el envejecimiento y la vejez es aún incipiente, tanto en la sociedad como en las políticas públicas de salud. El envejecimiento es complejo y no es necesario establecer límites ni fronteras entre estos dos aspectos, ni entre la salud y la enfermedad.

Palabras clave: Proceso salud-enfermedad; Envejecimiento; Anciano; Políticas públicas de salud.

\section{Introdução}

O envelhecimento é considerado um processo contínuo, individual, universal e irreversível. Ele pode ser entendido como um fenômeno complexo, que ocasiona a perda gradual das reservas fisiológicas, um aumento do risco de adquirir doenças e um declínio geral na capacidade intrínseca do indivíduo. Mas a idade avançada envolve mudanças que vão além das perdas biológicas, podendo incluir mudanças sociais, psicológicas e ambientais (Organização Mundial da Saúde, 2015; Pelegrino, 2009).

Cabe destacar que o envelhecimento é um processo que ocorre por toda a vida, do nascimento até a morte, enquanto a velhice é demarcada como a última fase da vida (Costa, Soares, 2016). A nível mundial e também no Brasil, o envelhecimento populacional têm sido motivo de preocupação, devido à modificação da pirâmide populacional e o aumento da população idosa e da expectativa de vida, e leva o tema para destaque e discussão no âmbito das políticas públicas, em face das implicações tanto no âmbito social como na saúde dessa população (Brasil, 2007; 2010; Organização Mundial da Saúde, 2015).

Assim, essas políticas vigentes evidenciam o marco cronológico para caracterizar o início da velhice - são considerados idosos pessoas com 60 anos ou mais no Brasil -, e acabam por caracterizar o "processo de envelhecimento" e a "velhice" como indissociáveis, voltando as políticas para essa parcela da população (Brasil, 2003; 2006; 2007; 2010; Organização Mundial da Saúde, 2015).

Mas o envelhecimento, reconhecido apenas no plano cronológico, pode ser falho e arbitrário, pois as mudanças que ocorrem não são lineares ou consistentes, e são apenas vagamente associadas à idade de uma pessoa em anos. Elas podem se manifestar em graus e momentos diversos, o que lhe traz uma característica heterogênea, dinâmica e de grande variabilidade tanto inter como intraindividual (Groisman, 2002; Pelegrino, 2009).

Além disso, o processo de envelhecimento traz dois conceitos comumente utilizados: a senescência, considerada o envelhecimento "normal", marcado por uma série de alterações inevitáveis no funcionamento do organismo, como o aparecimento de rugas na pele, redução celular e da função de alguns órgãos, mudanças na coloração dos cabelos e menor resistência física; e a senilidade, considerado o envelhecimento "patológico", quando esse processo vem acompanhado por uma condição de doença que ocasiona consequências e que requer intervenção (Brasil, 2007; Pelegrino, 2009).

Mas a complexidade envolvida no envelhecimento, e as experiências e vivências de cada indivíduo, seja no plano biológico, psicológico ou social, pode trazer uma confusão entre o que pode ser considerado um envelhecimento "normal" ou "patológico", principalmente no que se refere à "perdas" e "ganhos".

A velhice, ao mesmo tempo que pode ser marcada por perdas relacionadas ao processo de envelhecimento, como a diminuição da capacidade funcional, maior vulnerabilidade à doenças, pode ser uma fase em que a pessoa procura manter suas capacidades, autonomia e independência. Essa fase, aliada à experiência e sabedoria de vida, pode ser caracterizada por um processo ativo e de ganhos, com bem-estar e qualidade de vida (Brasil, 2015).

Frente a essas problemáticas, emergem algumas questões para reflexão: como ocorre o processo saúde-doença na velhice? O que pode ser considerado normal ou patológico? Como o processo saúde-doença no envelhecimento e na velhice é entendido no plano das políticas públicas de saúde, na perspectiva do normal e do patológico? Para tanto, o objetivo desse trabalho é: refletir acerca do processo saúde-doença na velhice, na perspectiva do normal e do patológico do filósofo Georges Canguilhem. 


\section{Metodologia}

O presente estudo possui natureza exploratória com abordagem qualitativa. Nesse tipo de abordagem, é importante a interpretação por parte do pesquisador sobre o fenômeno em estudo, seguindo um processo indutivo (Pereira et al., 2018). Assim, o artigo trata-se de ensaio teórico-reflexivo, com base filosófica, que se deu por meio da leitura da obra "O normal e o patológico", de Georges Canguilhem (2011). Este filósofo e médico francês defendeu sua Tese em 1943, em medicina, intitulada "Ensaio sobre alguns problemas relativos ao normal e ao patológico". Resumidamente, fazia uma crítica à visão de que o patológico seria uma variação quantitativa do normal, e de como a medicina estabelece o conceito de "normal", e por consequência o de "patológico", que se dá através de leis, normas, médias e padrões, e das ciências fisiologia e patologia (Canguilhem, 2011).

A partir das ideias do autor, pretende-se refletir acerca dos questionamentos apresentados na introdução, propondo dois capítulos: "o processo saúde-doença na velhice: o que é normal e o que é patológico?" e "envelhecimento e velhice no plano das políticas públicas de saúde”.

Para embasar a reflexão e a discussão, este ensaio conta ainda com o apoio de publicações científicas acerca das temáticas sobre envelhecimento humano, velhice, processo saúde-doença no envelhecimento e na velhice, normal e patológico, e algumas políticas públicas relacionadas à temática. Foi realizada leitura, análise e interpretação dos materiais buscando a reflexão e articulação com a obra citada, o tema e as questões propostas.

\section{Resultados e Discussão}

\subsection{O processo saúde-doença na velhice: o que é normal e o que é patológico?}

Um dos primeiros aspectos que emergem é o uso do critério cronológico como marco da velhice. Ele é utilizado na delimitação da população de estudos, análises epidemiológicas e com propósitos administrativos e legais no planejamento e execução de serviços e das políticas públicas (Santos, 2010; Duarte, 1999). Em países desenvolvidos, considera-se idosa a pessoa com 65 anos ou mais de idade; já nos países subdesenvolvidos, como o Brasil, é considerada idosa a pessoa com idade igual ou superior a 60 anos (World Health Organization, 2005).

O critério cronológico surge como um padrão, uma norma baseada em estudos populacionais e da expectativa de vida, que contribui para uma delimitação que a nível legal e social é muito eficiente, mas que quando se trata da individualidade de cada pessoa, pode tipificá-las e ser considerado falho (Canguilhem, 2011; Duarte, 1999).

Pessoas com a mesma idade cronológica podem estar em estágios completamente diferentes de envelhecimento (Groisman, 2002), como por exemplo: uma pessoa com 50 anos pode ter problemas na coluna e sua funcionalidade afetada, e sentir-se muito "velha" e mais próxima da morte, que uma pessoa com 80 , com ótimo vigor físico e intelectual, sem doenças crônicas e que ainda faz caminhadas pela manhã. Assim, não é porque uma pessoa cruza a linha dos 60 anos que passa a ter doença, perdas e dores.

A idade cronológica no processo de envelhecimento humano aparece, não só como uma normatividade vital, mas também social. Canguilhem nos fala que a vida média de um homem é relacionada com a ação que este exerce sobre si mesmo, no coletivo. Assim, o processo de envelhecimento, e consequentemente seu estágio final, a velhice e a morte, vão depender das condições não somente fisiológicas, mas sociais desse indivíduo (Canguilhem, 2011).

Pensando no processo de envelhecimento como um complexo, que envolve aspectos biológicos, psicológicos, sociais e ambientais, pode-se perceber que está longe de seguir um padrão rigoroso como a idade cronológica, mas que depende de muitas circunstâncias, como a genética, os modos de vida, a cultura, as escolhas, experiências e o percurso evolutivo de cada indivíduo, em sua polaridade dinâmica de vida, que vai determinar a heterogeneidade e individualidade desse processo, e as consequências, positivas ou negativas que vão ocorrer (Canguilhem, 2011; Schneider, Irigaray, 2008). 
Para tanto, outros critérios tem sido utilizados para definir o marco da velhice, como a preferência por falar de idade funcional, caracterizando o quão bem uma pessoa funciona em um ambiente físico e social em comparação a outras de mesma idade cronológica; idade social, marcando a capacidade de desempenho de papéis de uma pessoa na sociedade; idade biológica, demarcando as modificações físicas e biológicas que marcam esse processo; e idade psicológica, como função das modificações cognitivas e afetivas que produzem-se ao longo do transcurso temporal (Duarte, 1999; Schneider \& Irigaray, 2008).

Assim, o que vai definir "saúde", "doença", "normal" ou "patológico" depende, além desses aspectos, de um contexto onde o indivíduo está inserido, das experiências vividas, e da sua percepção subjetiva em relação ao processo de envelhecimento e à velhice.

Um dos aspectos influenciadores dessa percepção é que a sociedade (principalmente a ocidental) têm percebido o processo de envelhecimento como algo negativo, que traz doenças, incapacidades, e a velhice atrelada à proximidade da morte, sendo o idoso tratado como um empecilho, considerando, portanto, ambos como "patológicos". Ao contrário, sociedades não ocidentais reconhecem o lado positivo do envelhecimento e da velhice, os quais podem estar associados à aquisição de experiência e sabedoria (Uchôa, 2003; Guerra et al., 2021).

A ênfase da sociedade contemporânea na juventude e na beleza também faz com que o envelhecimento seja negado, e seja motivo de diversas intervenções (procedimentos estéticos, cirurgias, procedimentos de beleza) justamente para atingir os padrões normativos da sociedade. Há, aí, portanto, uma tentativa de "tratar" ou "deter" o envelhecimento por meio de uma normatização artificial, como se fosse algo naturalmente patológico (Canguilhem, 2011).

Pessoas idosas, através de uma vida ativa, uma boa auto-estima, e encarando o processo de envelhecimento e a velhice como positivos, conseguem ter uma ótima qualidade de vida, encarando os desafios impostos (Luiz et al., 2018). Mas o que tem de ser levado em conta é a individualidade e a adaptação de cada pessoa dentro dos seus limites e possibilidades, criando suas próprias normas de vida (Canguilhem, 2011).

Muitos indivíduos idosos, que apresentam doenças crônicas (hipertensão, diabetes, problemas cardíacos), certas limitações físicas, e que, dentro de um padrão fisiológico seriam considerados doentes, acabam por determinar nova ordem vital. Como diz Canguilhem (2011, p. 130): “a doença é, ao mesmo tempo, privação e reformulação", portanto, esses podem considerar-se como saudáveis e ter uma vida ativa e boa qualidade de vida tanto quanto uma pessoa que não tenha nenhuma doença. É uma nova norma trazendo a capacidade de criar condições de existência superando as normas anteriores.

Ao mesmo tempo, um indivíduo pode ter todos os "parâmetros normais", e, em decorrência do processo de envelhecimento, e principalmente por estar na velhice, considerar-se doente. Canguilhem (2011) afirma que existe uma necessidade da doença como uma maneira de se testar a saúde, ou até uma busca inconsciente da doença pelo homem. Talvez essa necessidade esteja ligada às transformações trazidas pelo envelhecer, que, para alguns indivíduos, pode causar sofrimento e angústia.

Canguilhem (2011, p. 143) afirma que "um velho saudável não é apenas uma ficção de poeta”. Percebe-se assim, que o que vai definir a saúde dentro do processo de envelhecimento e da velhice, é a possibilidade de cada indivíduo "ultrapassar as normas que definem o normal momentâneo, a possibilidade de tolerar infrações à norma habitual e de instituir normas novas em situações novas" (Canguilhem, 2011, p. 138). A "situação nova" vivenciada dentro da complexidade desse processo, vai ser muito relativa em cada indivíduo que a vivencia.

O que também acontece frequentemente, atrelado conjuntamente ao modelo biomédico de saúde, de racionalidade e de determinismo biológico, que visam o constante tratamento de doenças, é medicalização da velhice. Pessoas idosas, fazendo tratamentos ou usando medicamentos sem necessidade, tentativas de identificar, prevenir ou combater patologias que, muitas vezes, não existem, além de querer prolongar a vida humana (Cabrita \& Abrahão, 2014). 
Atrelado à cultura e influência das normativas da sociedade e do marco legal da velhice, as pessoas acreditam que, ao terem chegado nessa fase, estão fragilizadas ou adoecidas, adquirindo o hábito de consultar, procurar especialistas, e utilizar medicamentos e vitaminas, mesmo sem necessidade. É correto afirmar que podem ocorrer, na velhice, algumas situações específicas relacionadas ao processo de envelhecimento que precisam de intervenções e medicamentos, mas isso acaba sendo uma rotina, que é influenciada pela excessiva propaganda e incentivo ao consumo de medicamentos para a população nessa fase da vida (Vera, 2016).

A busca constante pela normalização do corpo, as imagens, a publicidade, o consumismo pela perfeição, também tratam de ditar o que é tido por normal e por consequência o que é patológico. Com tudo isso, confunde-se velhice normal e patológica. Segundo Canguilhem (2011), o médico persiste na abordagem das doenças, e por isso muitas vezes acaba por deixar de lado a percepção do indivíduo sobre si, o que para ele é considerado normal ou patológico.

O processo saúde-doença durante a velhice é extremamente complexo. O que é e quem define o que é normal e o que é patológico é a pessoa, e vai depender da vivência, das concepções e da construção social e contexto em que cada indivíduo está inserido, e principalmente, da sua individualidade e capacidade de estabelecer suas próprias normas.

Canguilhem (2011, p. 126) refere que "o limite entre o normal e o patológico torna-se impreciso", mas quando se trata do indivíduo, essa fronteira é precisa, pois é o próprio indivíduo quem sofre as consequências. Em se tratando da velhice, esse limite é mais confuso, tendo em vista todos os determinantes envolvidos nessa fase.

\subsection{Envelhecimento e velhice no plano das políticas públicas de saúde}

A Organização Mundial da Saúde (OMS), reconhecendo o envelhecimento populacional global, e as demandas sociais, políticas e econômicas advindas desse processo, evidencia a necessidade da criação de políticas de envelhecimento saudável e ativo (World Health Organization, 2005).

Assim, o envelhecimento saudável e ativo tem sido reconhecido para além da ausência de doenças ou simplesmente a perda de capacidades, sendo definido como o processo de desenvolvimento e manutenção da capacidade funcional que permite o bem-estar em idade avançada (Organização Mundial da Saúde, 2015). Ele é preconizado como um processo de otimização das oportunidades em saúde, aprendizagem ao longo da vida, participação e segurança, com o objetivo de melhorar a qualidade de vida à medida que o processo de envelhecimento ocorre e a velhice chega (Brasil, 2015; World Health Oganization, 2005).

Essa perspectiva do processo de envelhecimento, afastando a visão negativa de doença e de incapacidade, busca reconhecer a continuidade e a individualidade desse processo, em todas as esferas da vida de uma pessoa. Para tanto, reconhece que a perspectiva cronológica tem se tornado cada vez mais imprecisa, e que esse processo vai depender de um curso de vida que vai garantir (ou não) a chegada à velhice com bem-estar e qualidade, e também à longevidade.

A velhice, como última fase da vida, é vista como uma etapa vivenciada de forma heterogênea tanto individual como populacional. Por isso, o foco das concepções sobre o envelhecimento, por meio das recomendações das Nações Unidas e da OMS, visam o alcance da melhor qualidade de vida nessa fase, para que seja vivenciada com autonomia, independência, participação na sociedade e de forma positiva e holística (Brasil, 2015; Organização Mundial da Saúde, 2015).

A OMS traz uma visão sobre as especificidades de cada pessoa idosa, em que há fatores individuais (como hábitos de vida, mudanças relativas à idade, genética, doenças) e ambientais (como a moradia, tecnologias de apoio, equipamentos, transporte) que influenciam o processo saúde-doença na velhice, devendo, portanto, ser levados em consideração (Organização Mundial da Saúde, 2015).

Outra premissa dessas concepções, é que devem ser consideradas pessoas de todas as idades, incluindo pessoas frágeis, com alguma doença ou deficiência, assim como os idosos considerados saudáveis e ativos (Brasil, 2015; Organização Mundial da Saúde, 2015). Essa perspectiva de inclusão procura distanciar-se, de certo modo, das "normativas", buscando 
quebrar paradigmas atrelados ao envelhecer e à velhice, destacando implicitamente que o que é considerado "normal" ou "patológico" são normas individuais, e essa especificidade vai ditar o que vai ocorrer ao longo desse processo (Canguilhem, 2011).

No Brasil, o envelhecimento e a velhice têm sido considerados como prioridade nas agendas políticas, principalmente na área da saúde. A Política Nacional de Saúde da Pessoa Idosa é uma conquista nos direitos à saúde dessa população, tendo como finalidade a promoção, manutenção e recuperação da autonomia e independência desses indivíduos, direcionando medidas coletivas e individuais de saúde para este fim, e seguindo os princípios e diretrizes do Sistema Único de Saúde (SUS), e estando em acordo com as recomendações das Nações Unidas e a OMS (Brasil, 2006).

As políticas de saúde evidenciam, de certo modo, grandes mudanças em relação à como se vê o envelhecimento pela sociedade, trazendo novas concepções como o envelhecimento saudável e ativo, o reconhecimento da heterogeneidade e individualidade desse processo, e também da confusão da demarcação da velhice pela idade cronológica. Mas, ao mesmo tempo, o enfoque dessas políticas acaba sendo na própria velhice, demarcada, pelo "ser idoso".

Essa demarcação estaria padronizando a velhice, e o marco cronológico, tão criticado, é o que é levado em conta, independente se a pessoa com 50 anos considera-se velha, e a de 80 considera-se jovem. Assim, quem completa 60 anos automaticamente cruza a linha da velhice, como se precisasse involuntariamente de uma política, de um atendimento especial, de uma assistência, passando para um estado "patológico" (Canguilhem, 2011).

Além disso, os conceitos de "senescência", como envelhecimento "normal", e "senilidade", como envelhecimento "patológico" reforçam um caráter normativo, demarcando quem necessita ou não de assistência (Brasil, 2007). Um indivíduo pode, ao mesmo tempo, ter suas limitações e não considerar-se doente, e o contrário também é possível, como já foi discutido anteriormente. Assim, a heterogeneidade da velhice, sendo desconsiderada, reforça medidas normativas dentro das políticas (Mattar et al., 2019).

\section{Considerações Finais}

A quebra de paradigmas referentes ao envelhecimento e a velhice ainda é incipiente, tanto na sociedade quanto nas políticas públicas de saúde. Considerar o envelhecimento como algo heterogêneo, que é determinado por normas individuais, em que cada pessoa vive seu contexto, conhece seus limites e possibilidades dentro desse processo, é um dos caminhos para a mudança de concepções negativas referentes ao processo de envelhecimento e à velhice.

Há uma resposta para considerar o que é "normal" e o que é "patológico" dentro desse processo? Acredita-se que não, pois envelhecer é algo complexo, por isso fixar limites ou fronteiras entre essas duas vertentes, ou entre a saúde e a doença, é um recurso que não cabe aos profissionais de saúde, aos enfermeiros, ou a quem quer que seja, mas talvez a própria pessoa.

Ainda há a necessidade de estudos futuros, incluindo reflexões e pesquisas científicas, principalmente no que diz respeito à demarcação da velhice e a confusão sobre a visão do envelhecimento como um processo patológico e negativo pela sociedade. O presente estudo ainda pode contribuir para o melhor direcionamento das políticas públicas de saúde reforçando o caráter individual e específico de cada ser em seu processo de envelhecimento e de saúde-doença, na chegada à velhice.

\section{Referências}

Brasil (2003). Lei 10.741, de 1 de outubro de 2003. Dispõe sobre o Estatuto do Idoso e dá outras providências. Diário Oficial da União. Brasil (2006). Ministério da Saúde. Portaria nº 2.528 de 19 de outubro de 2006 - Aprova a Política Nacional de Saúde da Pessoa Idosa.

Brasil (2007). Ministério da Saúde. Secretaria de Atenção à Saúde. Departamento de Atenção Básica. Envelhecimento e saúde da pessoa idosa. Brasília-DF: MS. 192 p. 
Brasil (2010). Ministério da Saúde. Secretaria de Atenção à Saúde. Departamento de Ações Programáticas e Estratégicas. Atenção à saúde da pessoa idosa e envelhecimento. Brasília-DF. 44 p.

Brasil (2015). Centro Internacional de Longevidade Brasil (ILC-BRASIL). Envelhecimento ativo: um marco político em resposta à revolução da longevidade. $1^{\mathrm{a}}$ Ed. Rio de Janeiro: ILC-BRASIL. 119p.

Cabrita, B. A. C., \& Abrahão, A. L.(2014). O normal e o patológico na perspectiva do envelhecimento: uma revisão integrativa. Saúde em Debate, 38 (102), 635-645. doi: 10.5935/0103-1104.20140059.

Canguilhem, G. (2011). O normal e o patológico. (7a ed.), Forense-Universitária.

Costa, D. G. S., \& Soares, N. (2016). Envelhecimento e velhices: heterogeneidade no tempo do capital. Serviço Social \& Realidade, 25(2). https://ojs.franca.unesp.br/index.php/SSR/article/view/2519/2225

Duarte, L. R. S. (1999). Idade cronológica: mera questão referencial no processo de envelhecimento. Estudos Interdisciplinares sobre o envelhecimento, 2, 3547. $10.22456 / 2316-2171.5473$

Groisman, D. (2002). A velhice, entre o normal e o patológico. História, Ciências, Saúde — Manguinhos, Rio de Janeiro, 9 (1), 61-78. 10.1590/S010459702002000100004.

Guerra, M. F. S. de S., Porto, M. J., Araujo, A. M. B., Souza, J. P., Santos, G. P., Santana , W. N. B., Andrade, W. B., Santana, A. F., Silva, S. R. S., \& Nascimento , M. B. (2021). Envelhecimento: interrelação do idoso com a família e a sociedade. Research, Society and Development, 10 (1), e3410111534. $10.33448 /$ rsd-v10i1.11534.

Luiz, K. K. I., Loreto, M. D. D. S., Mafra, S. C. T., \& Ferreira, M. A. M. (2018). Envelhecimento e velhice: Protagonismo, temporalidade e desafios. Temporalis, 18 (35), 289-304. https://dialnet.unirioja.es/servlet/articulo?codigo=6580670

Mattar, J. B. L., Costa, D. G. S., Souza, C. F. S., Souza, E. B. P., \& Queiroz, A. D. L. S. (2019). A questão do envelhecimento: respostas políticoinstitucionais. In Congresso Brasileiro de Assistentes Sociais 2019 (Vol. 16, No. 1).

Organização Mundial da Saúde. (2015). Resumo do Relatório Mundial de Envelhecimento e Saúde. https://apps.who.int/iris/bitstream/handle/10665/186468/WHO_FWC_ALC_15.01_por.pdf

Pelegrino, P. S. (2009). Perspectiva biopsicológica do envelhecimento. São Paulo: Secretaria estadual de assistência e Desenvolvimento Social: Fundação Padre anchieta.

Pereira, A. S., Shitsuka, D. M., Parreira, F. J., \& Shitsuka, R. (2018). Metodologia da pesquisa científica. UFSM. https://repositorio.ufsm.br/bitstream/handle/1/15824/Lic_Computacao_Metodologia-Pesquisa-Cientifica.pdf?sequence=1.

Santos, S. S. C. (2010). Concepções teórico-filosóficas sobre envelhecimento, velhice, idoso e enfermagem gerontogeriátrica. Revista Brasileira de Enfermagem, 63(6), 1035-1039. 10.1590/S0034-71672010000600025.

Schneider, R. H., \& Irigaray, T. Q. (2008). O envelhecimento na atualidade: aspectos cronológicos, biológicos, psicológicos e sociais. Estudos de Psicologia, 25(4), 585-593. 10.1590/S0103-166X2008000400013.

Uchôa, E. (2003). Contribuições da antropologia para uma abordagem das questões relativas à saúde do idoso. Cadernos de Saúde Pública, 19 (3), 849-853. $10.1590 / \mathrm{S} 0102-311 \mathrm{X} 2003000300017$.

Vera, E. C. B. A. (2016). A medicalização do envelhecimento. https://www.portaldoenvelhecimento.com.br/medicalizacao-do-envelhecimento/

World Health Organization. (2005). Envelhecimento ativo: uma política de Brasília: Organização Pan-Americana da Saúde. 60p.

Zielinsky, I. M. B.(2008). Filosofia do envelhecimento: a dialética dos contrários. Revista Brasileira de Ciências do Envelhecimento Humano, 5(1). 